\title{
Una aproximación al análisis bioético del paradigma de las neurociencias en el estudio del autismo
}

\section{An approach to the bioethical analysis of the paradigm of neurosciences in the study of autistic disorder}

\author{
Melisa Corredera ${ }^{a}$ \\ anstituto de Investigaciones Económicas y Sociales del Sur. Bahía \\ Blanca, Buenos Aires, Argentina. \\ E-mail: melicorredera®gmail.com \\ María Belén Noceti ${ }^{b}$ \\ 'Instituto de Investigaciones Económicas y Sociales del Sur. Bahía \\ Blanca, Buenos Aires, Argentina. \\ E-mail: mbnocetiळgmail.com

\section{Fabio Hernán Álvarez ${ }^{c}$} \\ 'Universidad Nacional del Sur. Centro de Investigaciones \\ Bioéticas. Bahía Blanca, Buenos Aires, Argentina. \\ E-mail: fabiohalvarez.fløgmail.com

\section{Julieta de Battista ${ }^{d}$} \\ dUniversidad Nacional de La Plata. Facultad de Psicología. \\ La Plata, Buenos Aires, Argentina. \\ E-mail: julietadebattistaœgmail.com
}

\section{Correspondencia}

Melisa Corredera González

San Andrés, 800, Altos de Palihue, Bahía Blanca. Buenos Aires, Argentina. CP 8000 .

\section{Resumen}

Dada las peculiares características del autismo, la diversidad de sus formas y la gravedad a la que puede arribar este cuadro, profesionales e investigadores posicionados desde distintos paradigmas se ocupan por comprenderlo y detectarlo tempranamente. Cada paradigma define su campo de investigación y las metodologías que va a emplear para abordar el problema de investigación, sostenido y fundado en supuestos teóricos que explican la realidad y que están en consonancia con la comunidad científica a la que reconocen como legítima. Creemos que la forma en la que se comprende el autismo desde el paradigma de la neurociencia es un problema susceptible de ser abordado también desde la perspectiva bioética, en función a las conductas y las prácticas de los individuos que llevan a cabo las investigaciones en este campo. Se hace necesario profundizar sobre las implicancias bioéticas que tiene la aplicación de los avances tecnológicos y la introducción de máquinas e instrumentos en el estudio y experimentación con niños en búsqueda de la medición de sus manifestaciones corporales. Reflexionar desde una perspectiva bioética genera la posibilidad de introducir nuevos enunciados ante el posible riesgo de caer en posturas que objetualizan y reducen la complejidad del sujeto a un mero mecanismo, desestimando así como opera el concepto de niñez y la singularidad en cada caso. Palabras clave: Bioética; Autismo; Neurociencias; Niñez. 
Considering the peculiar characteristics of autism, the diversity of its shapes and the gravity of its diagnosis, researchers and professionals from different paradigms try to understand and detect the symptoms early. Each paradigm defines the field of research based on theoretical assumptions that explain reality and that are consistent with the scientific community recognised as legitimate. We believe that the understanding of autism according to the paradigm of neurosciences deserves also to be addressed from the bioethics perspective, regarding the behaviors and practices of those researchers working in this field. It is necessary to deepen into the bioethical implications of the application of technological advances and the introduction of machines and tools in the study and experimentation with children, in the attempt to measure body manifestations. Reflecting from the bioethics perspective provides the possibility of introducing new statements in the face of the possible risk of falling into positions that objectify and reduce the complexity of the child to a simple mechanism, thus dismissing how the concept of childhood and singularity operates in each case.. Keywords: Bioethics; Autistic Disorder; Neurosciences; Childhood.
En este trabajo analizaremos, desde la perspectiva de la bioética, las metodologías implementadas por científicos que adhieren al actual paradigma de las neurociencias en la investigación sobre el autismo (Cod.F.84.o CIE 10; Cod.29.oo DSM V) y sus implicancias en la praxis.

Dado que los actos son interpretados y juzgados como correctos o incorrectos en función del sistema de valores, de moral y justicia establecido mediante dispositivos institucionales por las costumbres de un grupo social (Douglas, 1986), para analizar algunas de las metodologías de indagación propias de este paradigma, nos posicionaremos desde la concepción de infancia y de los dispositivos institucionales que la definen desde el sistema jurídico en la República Argentina.

Creemos que la forma en la que se comprende el autismo desde la neurociencia es un problema susceptible de ser abordado desde la perspectiva bioética, en función de la aplicación de los avances tecnológicos para la medición de diversas manifestaciones corporales. En este sentido, pretendemos reflexionar sobre las conductas y las prácticas de los individuos que actualmente llevan a cabo las investigaciones dentro del campo del autismo desde el enfoque de las neurociencias.

Inicialmente presentaremos una descripción sobre el paradigma de investigación desde las neurociencias, ubicándonos en la temática del autismo con énfasis en las prácticas de medición de parámetros corporales. Procederemos a problematizarlas en un intento por acercarnos a responder las siguientes preguntas: ¿cuál es la concepción de sujeto y de infancia que subyace en las metodologías actuales de investigación sobre el autismo?, ¿qué rol juega el investigador en cada caso?, ¿cuál es el rol de los padres en relación al consentimiento informado?, ¿cómo se puede pensar desde esas experiencias el concepto de autonomía y autodeterminación?

\section{El paradigma de las neurociencias}

Un paradigma "es un conjunto de supuestos y prácticas reconocidas que, durante cierto periodo de tiempo proporcionan modelos de preguntas y 
soluciones a una comunidad" (Kuhn, 1971, p. 13). Desde cada paradigma se define el campo de investigación, el método y las metodologías que se van a emplear para abordar el problema de investigación, sostenidos y fundados en supuestos teóricos que explican la realidad y que están en consonancia con la comunidad científica reconocida para este paradigma. Belinchón (2013) afirma que se trata de "cosmovisiones completas muy complejas que se asientan sobre presuposiciones de muchas clases (modelos de sujeto, modelo de ciencia, modelos morales...) de las cuales apenas somos conscientes" (Vasen, 2015, p. 17).

Las neurociencias son un conjunto de disciplinas que tienen como objeto de estudio el sistema nervioso, y centran su interés en la forma en que la actividad del cerebro se relaciona con la conducta y con el aprendizaje. El Centro de Neurociencias, Mente y Comportamiento sostiene que el "principal objetivo de investigación en Neurociencia es ofrecer una comprensión mecanicista de la conducta de todo el organismo, un nivel de análisis más allá de las moléculas, células o circuitos individuales" (Salas Silva, 2003, p. 156).

En esa misma línea, desde el paradigma de las neurociencias, se comprende al autismo como un trastorno de origen neurobiológico que se manifiesta desde la temprana infancia y que se define en base a rasgos comportamentales. De esta forma de concebir y comprender el autismo se desprenden distintas metodologías que buscan profundizar sobre los fenómenos vinculados a esta problemática. El diseño de las metodologías, con las cuales se desarrollan diagnósticos y tratamientos, tiene rasgo fundamental y compartido con el paradigma positivista, la búsqueda de evidencia como forma de verificación de la verdad, único camino para la construcción de conocimiento.

Para conocer y comprender el funcionamiento del cerebro humano, las investigaciones en neurociencias implementan la aplicación de test y evaluaciones, así como también distintos desarrollos tecnológicos que permiten el registro y la medición de distintos parámetros corporales. Entre esas herramientas tecnológicas se destacan aquellas que permiten registrar la actividad cerebral a través de la neuroimagen o de la electroencefalografía, o sensores que brindan información sobre los movimientos del cuerpo asociados a las funciones cerebrales, entre otros.

Los niños con autismo se caracterizan por presentar trastornos en la interacción social, en la comunicación (expresión y comprensión del lenguaje), falta de flexibilidad mental, comportamientos, intereses y actividades estereotipadas y restringidas. Para obtener datos que permitan desentrañar la complejidad del funcionamiento cerebral de este cuadro, los investigadores posicionados en este paradigma, han desarrollado instrumentos que permiten medir distintos parámetros corporales. Algunos de esos instrumentos se basan en el estudio del seguimiento ocular de niños que presentan sospecha de padecer autismo o que ya fueron diagnosticados como autistas (Elison et al., 2013; Elsabbagh et al., 2012; Hosozawa et al., 2012; Shic; Macari; Chawarska, 2014). Estos estudios se valen de la utilización de softwares conocidos como eye trackers, («iMotions Eye Tracking Software», «EyeTracking Inc. -the eye tracking experts», «Innovative Eye Tracking Solutions»), que si bien son de extensa aplicación científica, algunos de ellos se han desarrollados específicamente para evaluar el punto donde el individuo con autismo fija la mirada o el movimiento de sus ojos en relación con su cabeza, buscando así identificar patrones de mirada alterados, tomando en cuenta los movimientos que realizan los ojos y la boca a partir de la interacción entre la persona y un ordenador. Los seguidores de ojos pueden trabajar a través de la luz, por lo general luz infrarroja, que se refleja en los ojos y se capta mediante una cámara de video u otro sensor óptico. La información recogida se analiza para extraer la rotación de los ojos y los cambios en los reflejos oculares. Los seguidores oculares más modernos usan el contraste para localizar el centro de la pupila y crear un reflejo de la córnea a través de la luz infrarroja. Para capturar el movimiento de los ojos existen diversos dispositivos: algunos de ellos toman la forma de vinchas y deben montarse en la cabeza del individuo estudiado; otros requieren que la cabeza se mantenga estable, y para ello se utiliza entonces un apoya-mentón. Además, existen dispositivos que siguen los movimientos de la cabeza de forma 
remota y automática, ubicando al individuo frente a una pantalla en la que se proyecta un video o se muestran imágenes de forma intermitente y en sus distintos espacios, registrando las respuestas oculares con una cámara web. Este tipo de herramineta busca detectar hacia dónde se dirige la mirada de bebés y niños para determinar regularidades en los patrones de comportamiento y así inferir si los niños estudiados padecen alguna de las condiciones asociadas al autismo.

Considerando la necesidad entonces de adaptar el cuerpo a dispositivos extra-somáticos de medición, los especialistas propician la posibilidad de que los individuos en estudio se familiaricen con estos elementos:

Antes de testear a los participantes, les mostramos el equipamiento y les damos tiempo para que se familiaricen con él, con las cámaras que se ubican en la cabeza y el procedimiento del testeo ocular. Los participantes son sentados a $8.40 \mathrm{~cm}$ frente al monitor, con sus cabezas estabilizadas a partir del uso de un dispositivo en el que apoyan la frente y la pera. Los movimientos del cuerpo, que pueden introducir inestabilidad en la grabación de los movimientos oculares, son controlados con el uso de un apoya pies y un apoyabrazos, además de una silla con respaldo que soporte los hombros y el torso. (Johnson et al., 2012, p. 3)

Otra línea de investigación desarrollada actualmente sobre la medición de parámetros corporales es aquella que se dedica al estudio de los movimientos del cuerpo, en especial a la relación que los movimientos guardan con el procesamiento mental. Para comprender la conexión entre los movimientos de las personas y sus pensamientos, desarrollan métodos para cuantificar el procesamiento mental de los individuos en relación con sus capacidades sensoriomotoras. Para la medición de los movimientos, se llevan a cabo experimentos en un laboratorio y se invita a participar a diferentes personas, las cuales, generalmente, al aceptar la invitación, reciben un pago por su contribución. Cuando el objetivo de la investigación es la indagación sobre el movimiento y los diferentes parámetros motores en el autismo, en el grupo de individuos seleccionados para el experimento se incluyen niños con dificultades en el desarrollo, que tengan sospecha de autismo, o que hayan sido diagnosticados como autistas, así como también niños con desarrollo típico para la comparación de los datos. Se persigue generar condiciones controladas dentro del laboratorio con el objetivo de medir las acciones de los individuos, las cuales reflejarían la naturaleza de su procesamiento mental, así como también darían cuenta de la predisposición que los individuos tienen para adquirir nuevos aprendizajes de manera racional o intuitiva. A partir de la medición y del análisis de los parámetros motores de estos pacientes, sería posible utilizar el sistema nervioso periférico como una entidad que permita desbloquear el control mental de los movimientos, y así lograr la conexión con el sistema nervioso central, propiciando modificaciones e induciendo cambios en el control central de las acciones de los niños (Torres et al., 2013).

Para la medición de los parámetros sensoriomotores, así como de las funciones respiratoria y cardíaca, y las señales electro encefálicas, los científicos cuentan con diferentes sensores instalados en dispositivos: vinchas, cascos, cinturones, o pulseras con abrojos o fajas, que contienen sensores y electrodos, y deben ser colocados en el cuerpo del individuo a estudiar. Una vez que esos dispositivos están instalados en el cuerpo, comienzan a registrar las funciones corporales y emitir señales que se almacenan en un procesador informático. ${ }^{1} \mathrm{El}$ experimentador brinda consignas al individuo y le ofrece diversos estímulos para evaluar su reacción y sus respuestas.

En función de lo mencionado, buscaremos profundizar sobre las implicancias bioéticas de la puesta en marcha de las metodologías descriptas y emergentes del enfoque neurocientífico en el estudio del autismo.

\footnotetext{
1 Para investigar y examinar los procesos de hibridación orgánico-tecnológicos, o la relación cuerpo-procesadores informáticos, se puede consultar Sibilia (2013).
} 


\section{Autismo: concepto y definición}

el autismo existe, pero no sabemos lo que es. Lynn Waterhouse

Entre los siglos XVI y XVII, el niño se concebía como un ser inacabado, incompleto, dependiente y limitado en su racionalidad. A partir del siglo XIX, con el surgimiento de los saberes vinculados al cuidado y al desarrollo de la infancia (estudios de pediatría, psiquiatría y psicología infantil, puericultura), se fue configurando un entramado normativo que estableció parámetros de normalidad y anormalidad infantil, direccionando en ese sentido las pautas vinculadas a la crianza y a la educación de los niños (Albarello; Manzione, 2015). De allí se deriva la tendencia de considerar la infancia como un período de la vida, una etapa que comprende, según Jaramillo (2007), desde la gestación hasta los 7 años aproximadamente, etapa de la que depende toda la evolución posterior del hombre.

Desde otro punto de vista, la legislación vigente en Argentina sostiene que la infancia es "una construcción del orden de lo social y simbólico que asume características propias del contexto sociohistórico, político y cultural en el cual surge [...] producto del desarrollo histórico humano" (Imhoff et al., 2011, p. 3). De ello se deduce que la característica principal de la niñez es la variabilidad de su contenido, según el tiempo y espacio sociocultural en el que se enmarque, coordenadas en las que la niñez es definida por cada cultura, a la que a su vez se le adjudican rasgos, roles y representaciones distintas (Ferran Casas, 1998). Por lo tanto, estableceremos que la propiedad general de la categoría niñez es ante todo la diversidad. (Noceti, 2008).

Dicha diversidad se ve amenazada frente a la actual medicalización y patologización de la vida (Mainetti, 1990), de la infancia, producto de las prácticas en salud mental relacionadas con el etiquetamiento y la producción de diagnósticos de manera expeditiva. ${ }^{2}$ El uso que se hace de la clasificación del autismo establecida en el Manual de Diagnóstico y Estadística de la Asociación Psiquiátrica Americana (2014) en su quinta edición (en adelante DSM V), es un ejemplo.

El DSM V fue editado en mayo de 2013, por la Asociación Estadounidense de Psiquiatría, y contiene una clasificación de los trastornos mentales, así como descripciones para cada categoría diagnóstica con la finalidad de que los médicos e investigadores de las ciencias de la salud puedan diagnosticar, estudiar e intercambiar información, así como tratar los distintos trastornos. Respecto a la problemática planteada en este trabajo, el DSM V define el término "espectro" a la amplia gama de signos, síntomas, habilidades y niveles de deterioro o discapacidad que pueden presentar los niños con este trastorno, y que van, desde un deterioro leve causado por sus síntomas, hasta condiciones con un nivel de discapacidad funcional grave. El DSM V permite identificar a los niños que presentan algunos o varios de los criterios allí enunciados, clasificando al TEA (trastorno del espectro autista) en función de su grado de severidad (Corredera; De Battista, 2016). Se trata de un enunciado de conductas que se agrupan en dos categorías de síntomas: (1) "deficiencias en la comunicación social" (los problemas sociales y de comunicación se combinan); y (2) "comportamientos restringidos y repetitivos". Dentro de cada una de esas categorías, se enumeran síntomas que ilustran con ejemplos y facilitan la identificación de rasgos patológicos en la conducta del niño. Según la puntuación de esos ítems, se determina el diagnóstico y su grado de severidad. Desde esta perspectiva, existen además otras herramientas para el diagnóstico, como encuestas a padres, test de evaluación semi-estructurados (SCQ) (Rutter; Lord, 1999); ADOS-G (Lord et al., 200o); ADI-R, (Le Couteur; Lord; Rutter, 2003), entre otros, y su aplicación busca determinar la presencia de comportamientos relacionados o compatibles con el

2 El etiquetamiento y la clasificación provienen de un saber médico que articula las variables etiológicas dentro de un modelo ontológico. El modelo ontológico asienta sus bases epistémicas en la medicina de las lesiones o anatómica, la medicina de las especies, o esencialismo, y la medicina de las especificidades. El modelo ontológico busca tipificar, discriminar, clasificar bajo la perspectiva de que la alteración funcional, la expresión sintomática y la alteración orgánica se corresponden (la patología se da en un lugar). El ser de la enfermedad se traduce en síntomas y tiene una causa precisa, específica, única (Laplantine, 1999). 
TEA, tanto en niños con sospecha o riesgo potencial de padecer autismo, como en la población de niños sanos, revistiendo así la posibilidad de ser, además, herramientas de prevención.

Vasen alerta sobre los riesgos que presenta la codificación del TEA: "el TEA incluye una enorme diversidad. [...] Dicha diversidad es de cuadros y de síntomas diversos en lo que aparece construido como el mismo cuadro. El espectro engloba clasificatoriamente, simplifica la codificación administrativa y homogeneiza clínicamente" (Vasen, 2015, p. 83).

Así, una enorme porción de chicos englobados dentro del TEA [...] provienen en nuestro país de familias con una inserción social precarizada, producto de los resabios de la debacle del empleo y la inserción social posterior a las políticas neoliberales. [...] Los modos de inscripción de los niños en la vida y en las fantasmáticas parentales no están al margen de esas dimensiones socioculturales y epocales. [...] Son chicos que deberían ser englobados con Problemáticas Complejas en la Infancia. (Vasen, 2015, p. 88-89)

Si bien se conoce que el proceso para arribar a un diagnóstico no es sencillo y requiere de varias instancias de observación y análisis de la conducta del niño, así como entrevistas con sus padres o cuidadores, en algunas ocasiones, el ejercicio del diagnóstico apresurado viene impulsado por la necesidad de asegurar al paciente el acceso y cobertura de los tratamientos. Tener un diagnóstico, que se encuentre codificado y nombrado en los manuales de diagnóstico reconocidos por la comunidad médica, facilita en Argentina, a través de la Ley de Sistema de Protección Integral de los Discapacitados (Ley $\mathrm{n}^{\circ}$ 22.431), la obtención del certificado de discapacidad, puerta de entrada, legalizada, libre y gratuita, a los tratamientos. ${ }^{3} \mathrm{~A}$ partir de estas acciones, que tienen como principal motor la intención de ayuda al niño y la familia, se termina promoviendo el etiquetamiento y la expansión medicalizadora (Vasen, 2015).

\section{La cuestión de lo normal y lo patológico}

\author{
Antes de que pueda medicalizarse la \\ "anormalidad", el comportamiento tiene que ser \\ definido y reconocido como anormal \\ Conrad en Ingleby
}

Para Canguilhem (1971), referirse a lo normal implica trabajar con un concepto dinámico y polémico. Explica que una norma es una regla, algo que endereza, que se propone como "posible modo de unificación de una diversidad, de reabsorción de una diferencia" (Canguilhem, 1971, p. 187). Mary Douglas refiere a la norma como la expresión sintética de la institución, en este caso la institución médica. Las instituciones definen lo idéntico, homogenizan una suerte de elementos heterogéneos mediante operaciones de analogía, creando la ilusión de que ciertas categorías son de índole natural, por tanto universal e incuestionables. Sumándose a los procesos de clasificación, "las disciplinas portan un discurso que será el de la regla: no el de la regla jurídica derivada de la soberanía, sino el de la regla natural, vale decir de la norma" (Foucault, 2006, p. 45).

Pero ocurre que, además del juicio médico, cobra peso la percepción que los pacientes tienen sobre sí mismos y las ideas dominantes que circulan en la sociedad en la que se encuentran inmersos, lo que impulsa la denominación de la "enfermedad" y el "estar enfermo" (Canguilhem, 1971). ${ }^{4}$

En el caso del autismo, identificamos la influencia y el impacto que tienen sobre la sociedad los discursos médicos que portan la publicidad: el día internacional del autismo o las campañas de difusión sobre la importancia de la detección precoz, son un ejemplo de ello. Pese a que por un lado colaboran con la prevención y el acceso a la

\footnotetext{
3 "La Constitución Argentina en su artículo 13 establece la salud como derecho del pueblo y deber del Estado. La República Argentina es un Estado Federal, integrada por 23 provincias y la Ciudad Autónoma de Buenos Aires (Capital Federal). Estas 24 unidades políticoadministrativas son las que tienen, por mandato constitucional, la responsabilidad del cuidado y la protección de la salud de la población. Como la salud es un derecho constitucional, se puede afirmar que no existen barreras legales que impidan el acceso de los argentinos a los servicios públicos de salud. Por eso es que se considera que éstos brindan una "cobertura universal'” (EL SISTEMA..., 20o6).

4 MAINETTI, J. Ensayo de biofilosofía, comunicación personal, por José Alberto Mainetti el 15 jul. 2010.
} 
atención, como contrapartida instalan ideas respecto a una epidemia de autismo, brindando datos que no responden a la realidad de nuestro país, así como también reduciendo en pocas frases la complejidad de dicho padecimiento.

Tener una patología confronta al sujeto con el ideal de normalidad y los valores que de ella se derivan, valores que, como se dijo anteriormente, se configuran como representaciones sociales según el grupo en el que el sujeto se encuentre inmerso. La cuestión de "obtener un diagnóstico, una etiqueta, legitima la gravedad del cuadro y la pertinencia de cierto tipo de abordajes terapéuticos, dado que se trata de un cuadro mayoritariamente difícil que requiere múltiples intervenciones" (Vasen, 2015, p. 9o). De allí se desprende una oferta de tratamientos y profesionales que mueven un negocio (Mainetti, 1990, p. 55) entorno al autismo, debido a que el hombre va a luchar para trasformar aquello que le representa un obstáculo y que le impide alcanzar el ideal de la vida, el "ser normal”. Estar enfermo

es un concepto de no valor, que comprende a todos los valores negativos posibles [...] estar enfermo significa ser perjudicial o indeseable o socialmente desvalorizado, etc. [...] Lo que es deseado como valor es la vida, una larga vida, la capacidad de reproducción, la capacidad de trabajo físico, la fuerza, la resistencia a la fatiga, la ausencia de dolor, un estado en el cual se note lo menos posible al cuerpo fuera del gozoso sentimiento de existencia. (Canguilhem, 1971, p. 88)

En ese contexto surge la oferta de los diferentes y numerosos tratamientos, con el objetivo de corregir o modificar aquellos comportamientos que apartan al sujeto de la norma y lo vuelven indeseable, clasificándolo y encasillándolo según su nivel de severidad.

Basándonos en el respeto por la diversidad e individualidad, proponemos la denominación de signos clínicos de autismo, ya que si bien existen características similares entre los niños con severos bloqueos en el desarrollo, consideramos que la subjetividad siempre se encuentra en proceso, en movimiento, y hablar del niño autista implicaría cerrarlo en categorizaciones que obturan la oportunidad de preguntarse, cuestionarse y crear nuevas intervenciones, obstruyendo a su vez las posibilidades en el despliegue de la subjetividad. Sostener la mirada y la escucha en la individualidad de cada sujeto evita que caigamos en simplificaciones o recitados de características sintomatológicas (Corredera; De Battista, 2016). Desde esta concepción, el tratamiento y el abordaje de niños que presentan signos clínicos de autismo requiere un diseño particular, un trabajo artesanal que se adecue a las necesidades individuales de cada uno de ellos. El terapeuta, rompiendo con los criterios de eficiencia, debe posicionarse como un artesano que va reorganizando, rediseñando las intervenciones mientras transcurre el tratamiento de su paciente. Por lo tanto, desde esta perspectiva no habría una receta o instructivo de tratamiento, solo ejemplos de intervenciones que pueden ser modificadas y adaptadas a las particularidades de otros niños.

\section{La cuestión de la tecnología y su implementación: aproximación a un análisis bioético}

Las formas de conocer e indagar un fenómeno encuentran su fundamento a partir de una perspectiva ética siempre en construcción en base a saberes y poderes en pugna. A partir de ese paradigma se definirá el tipo de relaciones que se van a establecer entre el investigador y su objeto de estudio. Los actos que este lleve a cabo estarán determinados por la moral vigente, propia del contexto en el que el investigador se encuentra inmerso, es decir, reglas de acción, fundadas en valores morales que orientarán su obrar.

Sobre la base de este planteo, en el siguiente apartado pondremos el foco y nos aproximaremos a una reflexión bioética respecto al uso e implementación de recursos tecnológicos para la medición de los parámetros corporales en los niños que presentan signos clínicos de autismo. Hemos descripto anteriormente algunos de los dispositivos diseñados y utilizados para tales fines, por lo cual, en esta instancia, buscaremos identificar las premisas que fundamentan la utilización de esos recursos 
tecnológicos y confrontarlas, por ejemplo, con el principio bioético de la autonomía ${ }^{5}$ y con ciertos elementos éticos que también intervienen en el ámbito bioético.

En el ámbito de las investigaciones con seres humanos, los científicos son situados, por el colectivo social, en un lugar de autoridad respecto a lo que saben y conocen sobre la problemática que afecta a los individuos estudiados, además de representar para ellos y sus familias la posibilidad de encontrar, a partir de la investigación, respuestas para las dificultades que trae aparejado su padecimiento.

Comenzaremos revisando la posición en el que los niños son ubicados dentro de estas experiencias de investigación.

Como se mencionó en el apartado anterior, los niños que presentan signos clínicos de autismo, manifiestan dificultades en la comunicación e interacción social; muchos de ellos no han logrado desarrollar el lenguaje verbal, por lo cual resulta difícil decodificar sus necesidades y deseos. También se señaló que sus comportamientos son repetitivos, muestran resistencia frente a los cambios de hábitos y espacios, además de experimentar, en la mayoría de los casos, un registro particular de las sensaciones corporales (registro interoceptivo y exteroceptivo), que se evidencian en los movimientos estereotipados o en la manipulación bizarra de ciertos objetos. Frente a la caracterización de su conducta, resulta difícil imaginar la implementación de las metodologías desarrolladas para el registro de los movimientos oculares y otros parámetros motores, ya que, en la mayor parte de las investigaciones, se requiere de la colocación de sensores en distintas partes del cuerpo del niño, que debe portar hasta la finalización de la experiencia o de la fijación de su cabeza en dirección a una pantalla para el seguimiento de sus pupilas.

Entendemos que en esas instancias el niño podría verse reducido y ubicado en el lugar de objeto de investigación, ${ }^{6}$ como un mecanismo cuantificable. El investigador, distanciándose afectivamente, lo dejaría relegado a sensaciones desagradables que los padres buscarían atenuar o controlar para lograr la concreción del experimento.

Estas prácticas son posibles de asociar a una concepción adulto-céntrica sobre la infancia, en la cual los padres o cuidadores responsables asumen al niño como una propiedad familiar, ligado a la cuestión filial. El niño es inmaduro, incapaz de decidir por sí mismo, dependiente del cuidado del adulto, el cual tiene la madurez y la experiencia para decidir lo mejor y más conveniente en cada caso. Dado que en los pacientes con signos clínicos de autismo no es posible referirse a cuestiones de consentimiento o conciencia respecto a la participación o no de un experimento, el niño está bajo el dominio de sus padres o cuidadores, quienes juzgarán por él y tomarán la decisión respecto a su participación. Entendemos que la participación representa para la familia la posibilidad de encontrar respuestas y soluciones al padecimiento de su hijo, factor que propicia la aceptación en la participación de la experiencia, a la vez que se ven seducidos cuando existe una remuneración de por medio que motiva a los sujetos a formar parte de la investigación.

La Declaración Universal sobre Bioética y Derechos Humanos (Unesco, 2005, p. 7) menciona: "Se habrá de respetar la autonomía de la persona

5 El principio de autonomía es uno de los cuatro principios que surge del Informe Belmont. Los principios bioéticos se utilizan como criterios para decidir y obrar dentro de la moral biomédica norteamericana (Beauchamp; Childress, 1994). Un paradigma ético biomédico que luego se extendió a otros contextos socio culturales. Nacida en el contexto de los EEUU, la teoría de los cuatro principios ha sido muy discutida en el contexto latinoamericano. Preferentemente porque desde esta teoría se puede generar un pragmatismo y procedimentalismo vacío; es decir, que no permita evaluar la complejidad y particularidades de cada caso o el entramado de fuerzas que hacen a la situación. La crítica latinoamericana puso en entredicho la moral vigente que se importó de los EEUU, al señalar que los derechos humanos no pueden codificares en el lenguaje de los cuatro principios (Tealdi, 2008). También habría que hacer una distinción entre planteos clásicos de la bioética, que presentan un análisis fundado en la autonomía, el conflicto de derechos y el paternalismo médico; y planteos alternativos, que presentan un análisis de las situaciones introduciendo consideraciones socio culturales, económicas, de género, etc., y muestran la variabilidad y la complejidad de los problemas (Luna; Salles, 2008).

6 Desde la Revolución Científica (s. XVI y XVII), con la división cartesiana de mente-cuerpo o res cogitans y res extensa, y con la perspectiva anatomista que se inicia con Vesalio, el cuerpo comienza a separarse de la persona, a ser "lo otro", como una especie de cosa o residuo. Este esquema abonó una medicina, aún en nuestros días, que solo se ocupa del cuerpo-cosa u objeto y deja de lado una mirada integral de la persona. Para este tema se puede ver Le Breton (2002, p. 10 y ss). 
respecto a la facultad de adoptar decisiones, asumiendo la responsabilidad y respetando la autonomía de los demás. Para las personas que carecen de la capacidad de ejercer su autonomía, se habrán de tomar medidas especiales para proteger sus derechos e intereses”.

En las situaciones planteadas, son los “mayores", padres, maestros, profesionales, jueces, entre otros, quienes conocen cuáles son las necesidades de los niños y las niñas, y qué es para ellos lo más conveniente, lo que muchas veces puede acarrear, de forma invisibilizada, fenómenos de manipulación y dominación [...], y que evidencia la existencia de una relación jerárquica y asimétrica con los adultos; son los adultos quienes deciden acerca de la vida y proyecto de los niños y las niñas, convirtiéndolos en objeto de intervención de profesionales, instituciones y políticas sociales y judiciales (Imhoff et al., 2011). Este aspecto se confronta con lo que se establece a partir de la Convención Internacional de los Derechos del Niño, donde se produce un cambio significativo en la forma de concebirlo. Desde esta convención, el niño/a deja de ser visto como objeto de derechos para comenzar a ser entendido como sujeto de una amplia gama de derechos y libertades, sosteniendo la igualdad social de todos/as. Así, el Paradigma de protección integral de la infancia, tiene como uno de sus objetivos primordiales "el interés superior del niño", lo cual implica que este debe ser considerado en todo lo que concierne a la toma de decisiones e implementación de acciones enfocadas a preservary promover su desarrollo físico, psicológico, educativo, cultural, moral, espiritual y social sin discriminación de ningún tipo (Imhoff et al., 2011, p. 16). En las investigaciones mencionadas, se pone en juego el accionar de los padres o cuidadores responsables del niño frente al principio de autonomía y al derecho a ser oído y atendido, derechos expuestos en los artículos $n^{0} 2$ y 3 de la Ley de Protección Integral de los Derechos de las niñas, niños y adolescentes -Ley no 26.061 (Argentina, 2005). Entonces cabe preguntarnos, ¿Cuál es el grado de beneficio para estos pacientes al participar de esas experiencias de investigación? Desde la lógica del utilitarismo podría fundamentarse que la incomodidad o malestar de los niños que participen en los experimentos estaría justificado por la posibilidad de hallar datos significativos que revelen nuevas formas de tratar las dificultades que trae aparejado este cuadro, en otras palabras, "la mayor utilidad para el mayor número”. Esta hipótesis resultaría insuficiente desde el concepto de dignidad humana o desde el concepto de acto justo. El utilitarismo no da cuenta del hecho de que frecuentemente valoramos ciertos actos porque son justos y no solo porque maximizan la felicidad o el mayor bien para el mayor número (Luna; Salles, 2008).

En la Fundamentación de la metafísica de las costumbres, Kant (1921, p. 42) propone: "Obra de modo tal que uses a la humanidad, tanto en tu persona como en la persona de cualquier otro, siempre y al mismo tiempo como fin y nunca simplemente como medio". Se problematiza desde este planteo la cuestión de la dignidad del hombre, su objetivización. El enajenamiento y distanciamiento afectivo derivaría de la necesidad de los investigadores por ajustarse a los criterios de verdad avalados como el único camino posible para la construcción de enunciados validados científicamente, lo que los conduciría a seleccionar aquellas metodologías que revisten un alto grado de verificabilidad y objetividad. Esta premisa deja de lado la influencia y las relaciones que se establecen entre el sujeto y el contexto de la investigación: "la simplicidad metodológica que supuestamente respalda el consejo utilitarista de limitarse a las inferencias "medios-fines" en el ejercicio de la razón práctica puede deslizarse con toda naturalidad por la pendiente de un indeseado reduccionismo" (Llueca Ortiz, 2013, p. 59). Al respecto, Canguilhem afirmaba:

es imposible negar la dificultad que existe para asimilar a condiciones experimentales las condiciones normales, tanto en el sentido estadístico como en sentido normativo, de la vida de los animales y del hombre [...] hay que decir que las condiciones de examen en el laboratorio colocan al ser vivo en una situación patológica, de la cual paradójicamente se pretende extraer conclusiones que tengan alcance normativo. (Canguilhem, 1971, p. 109)

Si es lícito definir el estado normal de un ser vivo como una relación normativa de ajuste al medio ambiente, es preciso no olvidar que el propio 
laboratorio constituye un nuevo ambiente. [...] Es imposible que los procesos de la vida en el laboratorio no retengan algún rasgo específico de su relación con el lugar y con el momento del experimento. (Canguilhem, 1971, p. 111)

En este sentido, la objetividad respecto al criterio de verdad se ve cuestionada. Los datos obtenidos están circunscriptos a las circunstancias particulares del experimento, volviéndose incierta la aplicación de los resultados a la población de niños que presentan signos clínicos de autismo. "La verdad del conocimiento científico siempre resulta falsable, y la verdad revelada solo dicen tenerla las religiones. Solo podemos alcanzar perspectivas parciales o medias verdades" (Tealdi, 2008, p. 202). De esta manera, sostenemos que las metodologías de investigación deben considerar al niño como un sujeto de derechos, y ese principio debe reflejarse en las prácticas y técnicas a partir de las cuales se aborda al sujeto para su indagación.

\section{Conclusiones}

Por un lado, y en función del análisis realizado, insistimos en resaltar que las redes de poder están plagadas de resistencias, insurrecciones, coágulos, contradicciones y líneas de fuga. Es por eso que el biopoder necesita beber constantemente en la fuente de los nuevos saberes y desarrollos tecnológicos para efectuar ajustes en sus dispositivos que le permitan fagocitar nuevos espacios vitales. Pero lejos de ser omnipotente, el biopoder tiene grietas a través de las cuales las fuerzas vitales logran infiltrarse y reaccionar. Aunque la vida esté sometida a cálculos explícitos y a otros controles, eso no significa que haya sido capturada íntegramente por técnicas capaces de dominarla y modelarla sin fisuras; al contrario, se escapa continuamente (Sibilia, 2013, p. 16o).

Por otro lado, pretendemos acentuar el lugar sustancial que el Comité de Bioética debería asumir en la revisión de los trabajos de investigación con seres humanos. Este comité debe propiciar instancias de discusión que permitan revelar las posiciones de poder, modular las fuerzas que se desprenden de esa asimetría, y reivindicar la autonomía y derechos de los sujetos. Observar las metodologías de investigación desde una perspectiva bioética genera la posibilidad de introducir nuevos enunciados, teniendo en cuenta cómo opera la singularidad en cada caso, es decir, la situación social, las condiciones socio económicas, y las condiciones de vulnerabilidad de los sujetos que son convocados a participar en cada investigación.

Parecería estar ausente el cuestionamiento y la reflexión sobre la introducción de máquinas e instrumentos tecnológicos en la experimentación con niños. En la propuesta de participación a estas experiencias, se solicita el consentimiento informado, suponiendo inicialmente un sujeto que, al firmar dicha documentación, podría posteriormente quedar abolido en el experimento mismo, objetualizado, maquinizado por la imperante necesidad de responder a las circunstancias del experimento. Además, cabe repensar la contextualización de las experiencias y su pertinencia en función de las características propias del cuadro estudiado.

Finalmente, ¿qué intereses se encuentran vinculados a la investigación sobre esta temática?; ¿es posible justificar el malestar de un individuo con el propósito de hallar beneficios para otros?; ¿cuál es el aporte real de las investigaciones sobre los parámetros corporales?; ¿de las experiencias llevadas a cabo en un laboratorio y las respuestas a partir del condicionamiento, se obtienen datos cercanos a la realidad del individuo?

Un gran eco nos grita: "el biopoder tiene grietas a través de las cuales las fuerzas vitales logran infiltrarse y reaccionar".

\section{Referencias}

ALBARELLO, L.; MANZIONE, A. Infancia

y educación. Espacios en blanco: Serie

indagaciones, Buenos Aires, n. 25, p. 97-103, jun. 2015. Disponível em: 〈https://goo.gl/eCsıbq>.

Acesso em: 30 jul. 2018.

APA - AMERICAN PSYCHIATRIC ASSOCIATION.

Manual diagnóstico y estadístico de los

trastornos mentales DSM-V-TR. Barcelona:

Masson, 2014. 
ARGENTINA. Ley n ${ }^{0}$ 26.061, de 28 de septiembre de 2005. Ley de protección integral de los derechos de las niñas, niños y adolescentes. Boletín Oficial, Buenos Aires, 28 oct. 2005. Disponível em: <https://goo.gl/gKZWWr〉. Acesso em: 30 jul. 2018.

BEAUCHAMP, T.; CHILDRESS, J. Principios de ética biomédica. New York: Oxford University Press, 1994.

BELINCHÓN, M. Todo sobre el autismo. Madrid: Alfaomega, 2013.

CANGUILHEM, G. Lo normal y lo patológico. Buenos Aires: Siglo XXI, 1971.

CORREDERA, M.; BATTISTA, J. Abordajes educativos y terapéuticos sobre las dificultades en la comunicación de niños con signos clínicos de autismo. Acta Psiquiátrica y Psicológica de América Latina, Buenos Aires, v. 62, n. 3, p. 195206, 2016.

DOUGLAS, M. Cómo piensan las instituciones. Madrid: Alianza, 1986.

EL SISTEMA de salud en Argentina. La Salud Como Derecho Social, [S.l.], 27 maio 2006.

Disponível em: <https://goo.gl/wZV3Ma>. Acesso em: 30 jul. 2018.

ELISON, J. et al. White matter microstructure and atypical visual orienting in 7-month-olds at risk for autism. The American Journal of Psychiatry, Washington, DC, v. 170, n. 8, p. 899-908, 2013. Disponível em: <https://goo.gl/jFy7E2>. Acesso em: 30 jul. 2018.

ELSABBAGH, M. et al. Infant neural sensitivity to dynamic eye gaze is associated with later emerging autism. Current Biology, Amsterdam, v. 22, n. 4, p. 338-42, 2012.

FERRAN CASAS, F. Infancia, perspectivas psicosociales. Barcelona: Paidós, 1998.

FOUCAULT, M. Defender la sociedad: curso en el Collège de France (1975-1976). 2. ed. Ciudad de México: Fondo de Cultura Económica, 2006.

HOSOZAWA, M. et al. How children with specific language impairment view social situations: an eye tracking study. Pediatrics, Itasca, v. 129, n. 6, p. e1453-e146o, 2012.

IMHOFF, D et al. Pensar la participación sociopolítica infantil a partir de los paradigmas de la infancia. In: SEMINARIO ELECTIVO NO PERMANENTE "PSICOLOGÍA POLÍTICA”, 2011, Córdoba. Ficha de Cátedra... Córdoba: UNC, 2011. p. 1-27. Disponível em: 〈https://goo.gl/NpxMjB〉. Acesso em: 30 jul. 2018.

JARAMILLO, L. Concepción de infancia. Revista del Instituto de Estudios Superiores en Educación, Baranquilla, n. 8, p. 108-123, 2007.

JOHNSON, B. P. et al. A closer look at visually guided saccades in autism and Asperger's disorder. Frontiers Integrative Neuroscience, Lausanne, v. 6, n. 99, p. 1-7, 2012.

KANT, I. Fundamentación de la metafísica de las costumbres. Madrid: RB Ediciones, 1921.

KUHN, T. La estructura de las revoluciones científicas. Buenos Aires: Fondo de Cultura Económica, 1971.

LAPLANTINE, F. Antropología de la enfermedad. Buenos Aires: Ediciones Del Sol, 1999.

LE BRETON, D. Antropología del cuerpo y modernidad. Buenos Aires: Nueva Visión, 2002.

LE COUTEUR, A.; LORD, C.; RUTTER, M. Autism diagnostic interview-revised. Los Angeles: Western Psychological Services, 2003.

LLUECA ORTIZ, E. Bioética personalista y bioética utilitarista. Cuadernos de Bioética, Murcia, v. 24, n. 1, p. 57-65, 2013.

LORD, C. et al. The autism diagnostic observation schedule-generic: a standard measure of social and communication deficits associated with the spectrum of autism. Journal of Autism and Developmental Disorders, New York, v. 30, n. 3, p. 205-223, 2000. Disponível em: <https://goo.gl/ w6LYkW>. Acesso em: 30 jul. 2018.

LUNA, F.; SALLES, A. Bioética: nuevas reflexiones sobre debates clásicos. Buenos Aires: Fondo de Cultura Económica, 2008. 
MAINETTI, J. Bioética fundamental: la crisis bioética. La Plata: Quirón, 1990.

MAINETTI, J. Ensayo de biofilosofía, 2010, comunicación personal, por José Alberto Mainetti el 15 jul. 2010.

NOCETI, B. Niñez en riesgo social y políticas públicas en la Argentina. Buenos Aires: EdiUNS, 2008.

RUTTER, M.; LORD, C. Autism screening questionnaire: diagnostic validity. British Journal of Psychiatry, Cambrigde, v. 175, p. 444-451, 1999.

SALAS SILVA, R. ¿La educación necesita realmente de la neurociencia? Estudios Pedagógicos, Valdivia, n. 29, p. 155-171, 2003.

SHIC, F.; MACARI, S.; CHAWARSKA, K. Speech disturbs face scanning in 6-month olds who develop autism spectrum disorder. Biological Psychiatry, Amsterdam, v. 75, n. 3, p. 620, 2014.

SIBILIA, P. Cuerpo, subjetividad y tecnologías digitales. Buenos Aires: Fondo de Cultura Económica, 2013.

TEALDI, J. (Comp.). Diccionario latinoamericano de bioética. Bogotá: Unesco: UNC, 2008.

TORRES, E. B. et al. Autism: the micro-movement perspective. Frontiers in Integrative Neuroscience, Lausanne, v. 7, p. 1-27, jul. 2013.

UNESCO - ORGANIZACIÓN DE LAS NACIONES UNIDAS PARA LA EDUCACIÓN, CIENCIA Y CULTURA. Declaración universal sobre bioética e derechos humanos. Paris, 2005. Disponível em: <http://bit.ly/2eJgYıp>. Acesso em: 30 jul. 2018.

VASEN, J. Autismo: ¿espectro o diversidad? Buenos Aires: Noveduc, 2015.

\section{Contribución de los autores}

Todos los autores contribuyeron equitativamente en la concepción y redacción del artículo.

Recibido: 07/05/2018

Aprobado: 14/06/2018 